\title{
Giant Magnetocaloric Effect in Manganites
}

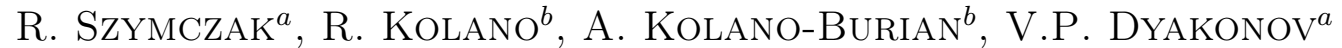 \\ AND H. SZYMCZAK ${ }^{a, *}$ \\ ${ }^{a}$ Institute of Physics, Polish Academy of Sciences, al. Lotnikq́o 32/46, 02-668 Warszawa, Poland \\ ${ }^{b}$ Institute of Non-Ferrous Metals, 44-101 Gliwice, Poland
}

\begin{abstract}
In this review we briefly discuss the magnetocaloric properties of the manganites. It is evidenced that the maximum entropy change observed in manganites exceeds that of gadolinium (the prototype material for room temperature refrigerators). For the manganites characterized by second-order magnetic phase transitions the scaling hypothesis is discussed. It is shown that simple phenomenological model with only one fitting parameter, presented in this paper, satisfactorily describes experimental observations.
\end{abstract}

PACS numbers: 75.47.Lx, 75.30.Sg, 75.40.Cx

\section{Introduction}

Recently, there has been a significant increase in research on the magnetocaloric effect (see review papers [1-7]). The development of a new magnetic refrigeration technology, based upon this effect, has brought an alternative to the conventional gas compression technique [1]. As a result, many new materials with large magnetocaloric effect have been discovered. The magnetocaloric effect is characterized by an adiabatic change in temperature (or an isothermal change in entropy, $\Delta S$ ) arising from the application of external magnetic field. The magnetocaloric effect arises due to the presence of two energy reservoirs in magnetic materials: one with phonon and the other with magnon excitations. These two reservoirs are coupled by the spin-lattice interactions. An external magnetic field affects the spin degrees of freedom resulting in heating or cooling of magnetic materials. This simple description of the magnetocaloric effect indicates that the highest change in temperature is expected for strongly magnetostrictive magnetic materials. The magnetocaloric effect increases with increase of the applied magnetic field and with the change of magnetization during application of magnetic field. This means that the effect reaches its maximum in the vicinity of magnetic phase transition points.

In this review we will briefly discuss the magnetocaloric properties of the manganites and the possibility of using these materials in magnetic refrigerators working near room temperature. It will be shown that the maximum entropy change observed in manganites is very high and exceeds that of gadolinium (the prototype material for room temperature refrigerators). In manganites ad-

* corresponding author; e-mail: szymh@ifpan.edu.pl vanced for active magnetic refrigerations paramagnetic-ferromagnetic transition is very narrow but no hysteresis is observed and the transitions are identified as second-order one. For these transitions the critical exponents are determined. Behaviour of the adiabatic magnetic entropy change in the critical region is discussed. In particular, the scaling hypothesis for magnetic entropy changes will be checked.

\section{General consideration}

The adiabatic magnetic entropy change $\Delta S_{H}$ of the magnetic system due to the application of a magnetic field $H$ is correlated with magnetization $M(T, H)$ by relation (see e.g. [1]):

$$
\Delta S_{H}(T, H)=\int_{0}^{H}(\partial M / \partial T)_{H} \mathrm{~d} H
$$

Concerning the magnetic transitions, one way of clarifying their nature is the determination of the critical exponents associated with them. Generally, the magnetization measurements are used to determine both magnetic entropy changes and critical exponents. It is well established that a continuous phase transition near $T_{\mathrm{C}}$, according to the scaling hypothesis [8], shows a power law dependence of magnetization $M(T)$ on reduced temperature $\varepsilon=\left(T-T_{\mathrm{C}}\right) / T_{\mathrm{C}}$ and magnetic field $H$ :

$$
\begin{aligned}
& M(T)=M_{0}(-\varepsilon)^{\beta}, \varepsilon<0 \\
& M\left(T_{\mathrm{C}}\right)=D H^{1 / \delta}, \varepsilon=0 \\
& H / M(T)=\left(h_{0} / M_{0}\right) \varepsilon^{\gamma}, \varepsilon>0, H \rightarrow 0
\end{aligned}
$$

The critical exponents $\beta, \gamma$ and $\delta$ are related through the Widom scaling relation [8]: $\beta \delta=\beta+\gamma$. The field dependence of the magnetic entropy change can be expressed as $\Delta S \sim H^{n}$ where $n=1+(\beta-1) /(\beta+\delta)[9]$.

The magnetic equation of state in the critical region may be written as 
$M(H, \varepsilon)=\varepsilon^{\beta} F_{ \pm}\left(H / \varepsilon^{\beta+\gamma}\right)$,

where $F_{+}$for $T>T_{\mathrm{C}}$ and $F_{-}$for $T<T_{\mathrm{C}}$, respectively, are regular functions. Using Eq. (5) one may determine behaviour of the adiabatic magnetic entropy change $\Delta S_{H}$ in the critical region:

$$
\Delta S_{H}(H, \varepsilon)=\varepsilon^{\beta} f_{ \pm}\left(H / \varepsilon^{\beta+\gamma}\right)
$$

The adiabatic magnetic entropy changes are also very often described in the frame of molecular field model [10].

The experimental results may also be analyzed in frames of a phenomenological statistical model developed by Biernacki [11-13]. It is a two-level model describing specific behaviour of the $e_{\mathrm{g}}$ electrons in manganites. The model contains two adjustable parameters derived from the experimental value of $T_{\mathrm{C}}$. Important element of the model is that it considers explicitly the electron-phonon interaction. According to the model, the free energy of the system can be expressed as

$$
\begin{aligned}
& F=\varepsilon n^{2}-\mu H \\
& \left.\quad-k_{\mathrm{B}} T \ln \left(+2 \exp \left(-(t R-2 \varepsilon n+2 \mu H) / k_{\mathrm{B}} T\right)\right)\right)
\end{aligned}
$$

where $n \in[0,1]$ is the $e_{\mathrm{g}}$ electron distribution function, $t R$ is the product of the hopping integral $t$ between $\mathrm{Mn}$ ions multiplied by the Huang-Rhys factor $R, \varepsilon$ is the Jahn-Teller energy, $\mu$ is the magnetic moment.

The electronic entropy $S$ can be obtained from Eq. (7):

$$
\begin{aligned}
& S(H, T)=-k_{\mathrm{B}} \\
& \quad \times[-n \ln 2+n \ln n+(1-n) \ln (1-n)] .
\end{aligned}
$$

The above expression is used to calculate the magnetic entropy change as a function of temperature and magnetic field with $T_{\mathrm{C}}$ taken as a fitting parameter.

The criterion of maximum $\left|\Delta S_{H}\right|$ is, of course, not the only one. According to [14] refrigerant capacity defined as $\mathrm{RC}=\left|\Delta S_{\mathrm{M}}\right| \Delta T_{\text {cyc }}\left(\Delta T_{\text {cyc }}=T_{\text {hot }}-T_{\text {col }}\right.$, where $T_{\text {hot }}$ and $T_{\text {col }}$ are the upper and the lower operating temperatures in the cycle) should reach its maximum value. Such defined parameter $\mathrm{RC}$ is a compromise between the magnitude of the magnetic entropy change and the width of the peak. Moreover, it is a suitable metrics for comparing the performance of different materials. However, the most important parameter for the magnetic refrigerators with a limited high magnetic field volume is the relative cooling power (RCP) $[15,16]$ defined as:

$\operatorname{RCP}(S)=\left|\Delta S_{H}\right| \delta_{\mathrm{FWHM}}$,

where $\delta_{\mathrm{FWHM}}$ is the full width at half maximum of the magnetic entropy change $\left|\Delta S_{H}(T)\right|$ curve.

All of the parameters related to magnetocaloric effect, such as the magnetic entropy change, refrigerant capacity and relative cooling power depend strongly on the magnitude of the applied magnetic field $H$. The knowledge of their field dependences is of great importance since it provides tools for making plausible extrapolations and interpolations to magnetic-field values necessary for construction of magnetocaloric refrigerators. Generally, the mentioned parameters increase with increasing maximum applied field $H$. The first explanation of the field dependence of $\left|\Delta S_{H}\right|$ was given by Oesterreicher and
Parker [17]. They have shown that in mean field approximation at the Curie temperature $\left|\Delta S_{H}\right| \propto H^{n}$, where $n=2 / 3$ (see also [18]). Franco et al. [9] have studied the field dependence of $\left|\Delta S_{H}\right|$ in materials with a second order phase transition in three characteristic regions: well below the Curie temperature $\left(T_{\mathrm{C}}\right)$, at $T_{\mathrm{C}}$, and well above $T_{\mathrm{C}}$. According to their calculations for temperatures well below $T_{\mathrm{C}}, n=1$ and well above $T_{\mathrm{C}}, n=2$. To determine $n\left(T_{\mathrm{C}}\right)$ the Arrott-Noakes equation of state [19] was used. It leads to the following expression:

$$
n\left(T_{\mathrm{C}}\right)=1+\delta^{-1}\left(1-\beta^{-1}\right),
$$

where $\delta$ and $\beta$ are the critical exponents. In the case of a mean field model $\delta=3, \beta=1 / 2$ and consequently $n\left(T_{\mathrm{C}}\right)=2 / 3$. In the same approximation, the relative cooling power depends on applied field $H$ according to the law $H^{n}$ with $n=1+\delta^{-1}$ [20]. Recently, it was shown [21] that besides the term $H^{2 / 3}$, a small negative term independent of $H$ influences $\Delta S_{H}(H)$ dependence. This term arises due to spatial inhomogeneity of real ferromagnetic materials.

\section{Magnetocaloric effect in manganites}

In recent years, there has been increasing interest in using manganites not only as materials with a colossal magnetoresistivity but also as materials with interesting magnetocaloric properties. The manganites with various compositions have been examined from this point of view (see $[5,7,22]$ for references). A large magnetocaloric effect makes manganites excellent candidates for working materials in magnetic refrigeration units especially because they are less costly than any other materials, particularly those based on Gd.

Recently, we have shown [23] that the changes in entropy near $T_{\mathrm{C}}$ depend strongly on various extrinsic factors. These dependences suggest that the magnitude of the magnetocaloric effect should depend strongly on methods of sample preparation. We have performed detailed studies of the magnetocaloric effect for $\mathrm{La}_{1-x} \mathrm{Ca}_{x} \mathrm{MnO}_{3}$ with $x=0.3,0.35$ and 0.4 , prepared by a nonstandard ceramic method. It was shown that the sharpness of the paramagnetic to ferromagnetic transition increases with $\mathrm{Ca}$ doping. This transition in $\mathrm{La}_{0.7} \mathrm{Ca}_{0.3} \mathrm{MnO}_{3}$ was shown to be extremely sharp suggesting the first-order phase transition. Nevertheless, we have evidenced that this manganite is characterized by a second-order transition near to the first-order one. In this manganite the magnetocaloric effect is very high (Fig. 1) and reaches $8 \mathrm{~J} /(\mathrm{kg} \mathrm{K})$ for $\Delta H=2 \mathrm{~T}$. It is the highest value ever observed for doped $\mathrm{LaMnO}_{3}$ manganites, larger than that known for gadolinium. The same compounds but in nanocrystalline form were studied in [24-26]. It was shown that value of $\left|\Delta S_{H}\right|$ in the nanocrystalline manganite is very small. However, the magnetocaloric effect for this manganite is spread over a remarkably broad temperature interval. For example, at $2 \mathrm{~T}$ magnetic field, the $\left|\Delta S_{H}\right|$ is equal to $0.32 \mathrm{~J} /(\mathrm{kg} \mathrm{K})$ corresponding to the level of full width at half minimum, 
but spreads over more than $80 \mathrm{~K}$. The analysis of the experimental data suggests the importance of magnetoelastic coupling in magnetocaloric properties of nanocrystalline manganites.

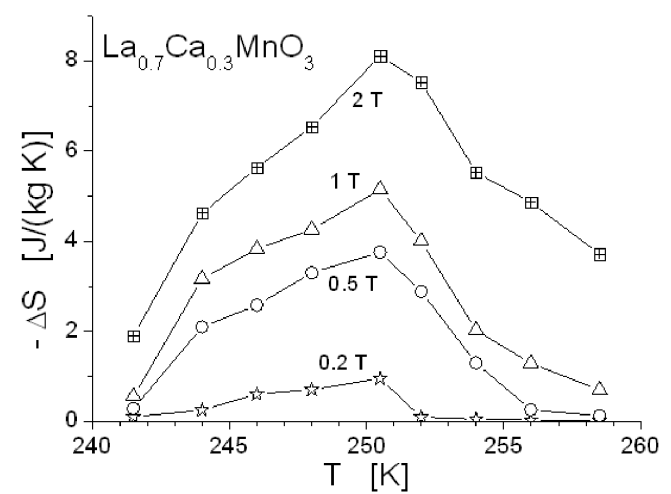

Fig. 1. Change of the magnetic entropy of $\mathrm{La}_{0.7} \mathrm{Ca}_{0.3} \mathrm{MnO}_{3}$ polycrystals as a function of temperature at fixed values of the external magnetic field.

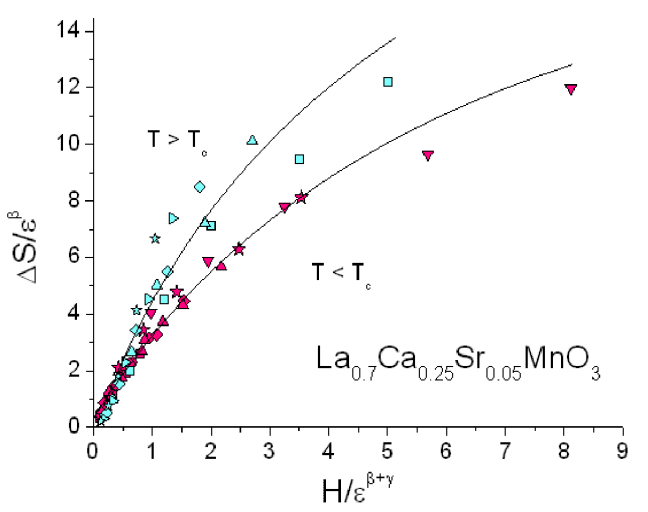

Fig. 2. A scaling plot of $\Delta S / \varepsilon^{\beta}$ vs. $H / \varepsilon^{\beta+\gamma}$ for $\mathrm{La}_{0.7} \mathrm{Ca}_{0.25} \mathrm{Sr}_{0.05} \mathrm{MnO}_{3}$.

Very high magnetocaloric effect was observed in $\mathrm{La}_{0.7} \mathrm{Ca}_{0.3-x} \mathrm{Sr}_{x} \mathrm{MnO}_{3-\delta}$ manganites [27, 28]. The origin of the large low-field $\left|\Delta S_{H}\right|$ in the $x \leq 0.05$ samples is associated with the metamagnetism driven by the oxygen deficiency in these samples. Promising candidates for the magnetic refrigerators around room temperature are La-based manganites doped with monovalent ions $\left(\mathrm{Na}^{+}\right.$, $\mathrm{Ag}^{+}, \mathrm{K}^{+}$) - see [29] and references therein.

In order to check whether $\Delta S_{H}$ measurements in the critical region obey the scaling relation given by Eq. (6), $\Delta S / \varepsilon^{\beta}$ as a function of $H / \varepsilon^{\beta+\gamma}$ is plotted in Fig. 2. It can be clearly seen that the experimental data fall on two curves, one for $T>T_{\mathrm{C}}$ and the other for $T<T_{\mathrm{C}}$. The results obtained are in agreement with the scaling hypothesis. It seems that a phenomenological statistical model developed by Biernacki [11-13] is also very effective. Figure 3 presents [30] the experimental

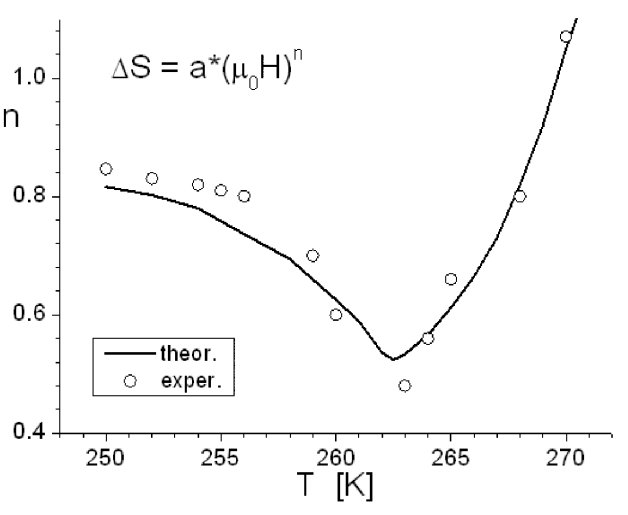

Fig. 3. Experimental and theoretical temperature dependences of the exponent characterizing the field dependence of $\Delta S_{\mathrm{M}}$ for the $\mathrm{La}_{0.6} \mathrm{Ca}_{0.4} \mathrm{MnO}_{3}$ manganite.

and theoretical temperature dependences of the exponent characterizing the field dependence of $\Delta S_{H}$ for the $\mathrm{La}_{0.6} \mathrm{Ca}_{0.4} \mathrm{MnO}_{3}$ manganite. It is seen that this simple model with only one fitting parameter satisfactorily describes experimental observations.

\section{Acknowledgments}

This work was financially supported by European Fund for Regional Development (Contract No. UDA-POIG.01.03.01-00-058/08/00).

\section{References}

[1] V.K. Pecharsky, K.A. Gschneidner Jr, J. Magn. Magn. Mater. 200, 44 (1999).

[2] A.M. Tishin, in: Handbook of Magnetic Materials, Vol. 12, Ed. K.H.J. Buschow, Elsevier, Amsterdam 1999, p. 395.

[3] K.A. Gschneidner Jr., V.K. Pecharsky, A.O. Pecharsky, C.B. Zimm, Rare Earth' 98 315-3, 69 (1999).

[4] A.M. Tishin, Y.I. Spichkin, The Magnetocaloric Effect and its Applications, Institute of Physics Publishing, Bristol 2003.

[5] K.A. Gschneidner Jr., V.K. Pecharsky, A.O. Tsokol, Rep. Prog. Phys. 68, 1479 (2005).

[6] E. Bruck, J. Phys. D, Appl. Phys. 38, R381 (2005).

[7] M.-H. Phan, S.-C. Yu, J. Magn. Magn. Mater. 306, 325 (2007).

[8] H.E. Stanley, Introduction to Phase Transition and Critical Phenomena, Oxford University Press, New York 1971.

[9] V. Franco, J.S. Biazquez, A. Conde, Appl. Phys. Lett. 89, $222512(2006)$.

[10] A. Szewczyk, H. Szymczak, A. Wisniewski, K. Piotrowski, R. Kartaszynski, B. Dabrowski, S. Kolesnik, Z. Bukowski, Appl. Phys. Lett. 77, 1026 (2000).

[11] S.W. Biernacki, Solid State Commun. 116, 471 (2000). 
[12] S.W. Biernacki, Phys. Rev. B 66, 094405 (2002); ibid 68, 174417 (2003).

[13] S.W. Biernacki, H.-J. Schulz, Phys. Rev. B 70, 092405 (2004).

[14] M.E. Wood, W.H. Porter, Cryogenics 25, 325 (1985).

[15] K.A. Gschneidner Jr., V.K. Pecharsky, Ann. Rev. Mater. Sci. 30, 387 (2000).

[16] J. Yang, Y.P. Lee, J. Appl. Phys. 102, 033913 (2007).

[17] H. Oesterreicher, F.T. Parker, J. Appl. Phys. 55, 4334 (1984)

[18] Q.Y. Dong, H.W. Zhang, J.L. Shen, J.R. Sun, B.G. Shen, J. Magn. Magn. Mater. 319, 56 (2007).

[19] A. Arrott, J.E. Noakes, Phys. Rev. Lett. 19, 786 (1967).

[20] V. Franco, A. Conde, J.M. Romero-Enrique, J.S. Blazquez, J. Phys., Condens. Matter. 20, 285207 (2008).

[21] M.D. Kuz'min, M. Richter, A.M. Tishin, J. Magn. Magn. Mater. 321, L1 (2009).
[22] M.-H. Phan, S.-C. Yu, Phys. Status Solidi A 204, 4091 (2007)

[23] R. Szymczak, M. Czepelak, R. Kolano, A. Kolano-Burian, B. Krzymanska, H. Szymczak, J. Mater. Sci. 43, 1734 (2008).

[24] M. Pekala, V. Drozd, J.F. Fagnard, P. Vanderbemdent, M. Ausloos, Appl. Phys. A 90, 237 (2008).

[25] W.J. Lu, X. Luo, C.Y. Hao, W.H. Song, Y.P. Sun, J. Appl. Phys. 104, 113908 (2008).

[26] C.L. Lu, K.F. Wang, S. Dong, J.G. Wan, J.-M. Liu, Z.F. Ren, J. Appl. Phys. 103, 07F714 (2008).

[27] A.N. Ulyanov, J.S. Kim, Y.M. Kang, D.G. Yoo, S.I. Yoo, J. Appl. Phys. 104, 113916 (2008).

[28] A.N. Ulyanov, Y.M. Kang, S.I. Yoo, J. Appl. Phys. 103, 07B328 (2008).

[29] W.C.-R. Koubaa, M. Koubaa, Cheikhrouhou, J. Mater. Sci. 44, 1780 (2009).

[30] R. Szymczak, A. Kolano-Burian, R. Kolano, R. Puzniak, V.P. Dyakonov, E.E. Zubov, O. Iesenchuk, H. Szymczak, Proc. E-MRS in press. 